\title{
离子液体/相转移催化剂促进的二茂铁基查尔酮的合成
}

\author{
赵海英* 尹凤楠 于玲岩李保国边占喜* \\ (内蒙古大学化学化工学院 内蒙古自治区精细有机合成重点实验室 呼和浩特 010021)
}

\begin{abstract}
摘要 乙酰基二茂铁与芳香醛在离子液体或相转移催化剂作用下进行 Claisen-Schmidt 缩合, 高产率地合成二茂铁基查 尔酮. 其中, 相转移催化剂能更有效地催化该反应, 优化后的反应条件为: 无水乙醇溶液, $35{ }^{\circ} \mathrm{C}, n$ (乙酰基二茂铁)： $n$ (芳香醛) $: n$ (四丁基六氟磷酸铵) $: n(\mathrm{NaOH})=1: 1: 2.5: 0.75$.

关键词 二茂铁; 查尔酮; 离子液体; 相转移催化剂; Claisen-Schmidt 缩合
\end{abstract}

\section{Facile Synthesis of Ferrocenylchalcone Promoted by Ionic Liquid or Phase-Transfer Catalyst}

\author{
Zhao, Haiying* Yin, Fengnan $\quad$ Yu, Lingyan $\quad$ Li, Baoguo $\quad$ Bian, Zhanxi* \\ (Inner Mongolia Key Laboratory of Fine Organic Synthesis, College of Chemistry and Chemical Engineering, \\ Inner Mongolia University, Hohhot 010021)
}

\begin{abstract}
The Claisen-Schmidt condensation of acetylferrocene with arylaldehydes in the presence of ionic liquids (ILs) or phase-transfer catalysts (PTCs) provided the corresponding ferrocenylchalcones in high yields, and PTCs can promote the condensation reaction better than those of ILs. Optimum conditions were as follows: molar ratio of acetylferrocene, arylaldehyde, $n-\mathrm{Bu}_{4} \mathrm{NPF}_{6}$ and $\mathrm{NaOH}$ is $1: 1: 2.5: 0.75$, and the appropriate reaction temperature is $35{ }^{\circ} \mathrm{C}$ in anhydrous EtOH.

Keywords ferrocene; chalcone; ionic liquid; phase-transfer catalyst; Claisen-Schmidt condensation
\end{abstract}

查尔酮作为优良的光敏材料具有潜在的非线性光 学性及液晶性, 被广泛用于光学及分子电子学等领 域 ${ }^{[1,2]}$. 查尔酮还是合成天然产物黄酮与异黄酮前体的 主要成分, 并且查尔酮本身也有重要的药理作用 ${ }^{[3,4]}$. 经 二茂铁修饰的查尔酮衍生物表现出较好的抗菌、抗寄生 虫、抗炎、抗血小板凝集等多种药理学性质 ${ }^{[57]}$. 因此, 对二茂铁类查尔酮化合物的研究与开发成为药物化学 的一个研究热点 ${ }^{[8]}$. 此外, 二茂铁查尔酮是重要的有机 合成中间体, 可以发生一系列重要的化学反应, 如迈克 尔加成反应 ${ }^{[9]}$, 从而合成多种杂环化合物, 如吡唑啉、异 噁唑以及含有两个以上的杂环化合物. 这些杂环化合物 具有较好的生物活性 ${ }^{[10]}$ 及电子发光性质 ${ }^{[11]}$.

鉴于二茂铁查尔酮化合物在诸多领域的应用, 如何 高效地合成二茂铁查尔酮及其衍生物成为合成化学中 的一个重要研究课题. 在多年的探索中, 各种方法曾被
尝试运用来合成查尔酩 ${ }^{[12]}$, 例如 Suzuki 反应、Witting 反应、肉桂酰氯的 Friedel-Crafts 酰基化、肉桂酸苯酯的 光照下重排反应等. 其中, Claisen-Schmidt 缩合反应是 最为行之有效的方法 ${ }^{[13]}$. 虽然二茂铁也属于芳香化合 物, 但是由于二茂铁的特殊几何及电子特性, 传统的这 些方法对于合成二茂铁衍生物往往并不适用, 例如在 $\mathrm{KOH}$ 或 $\mathrm{NaOH}$ 作用下乙醇溶液中通过 Claisen-Schmidt 缩合反应合成二茂铁查尔酮通常产率不高 ${ }^{[14 \sim 17]}$. 与传 统的方法相比, 微波辐射 ${ }^{[18]}$ 或超声促进 ${ }^{[19]}$ 是被公认的 清洁、低能、方便的方法，大量的有机反应运用这种技 术能高产率的得到相应的目标化合物，但是由于二茂铁 在微波炉中容易燃烧，这种方法往往不适用合成二茂铁 衍生物. 运用在玛瑙研钵中研磨的无溶剂条件进行乙酰 基二茂铁与部分芳香醛 Claisen-Schmidt 缩合反应可以 高产率的合成二茂铁查尔酮, 但是因为有些酫是液态,

\footnotetext{
*E-mail: hyzhao@imu.edu.cn, bzx1957@sina.com

Received November 25, 2015; revised December 14, 2015; published online January 15, 2016.

Project supported by the National Natural Science Foundation of China (No. 21562032), the Research Program of Science and Technology at Universities of Inner Mongolia (No. NJZZ001) and the Natural Science Foundation of Inner Mongolia (Nos. 2013MS0207, 2014JQ02).

国家自然科学基金(No. 21562032)、内蒙古自治区高等学校科学研究(No. NJZZ001)和内蒙古自治区自然科学基金(Nos. 2013MS0207, 2014JQ02)资助 项目.
} 
在该条件下容易挥发, 不适用某些二茂铁查尔酮 ${ }^{[20 ~ 22] . ~}$ Toma $^{[23]}$ 和 Villemin 等 ${ }^{[24]}$ 分别用相转移催化剂 18-crown6 和季铵盐 A336 催化 Claisen-Schmidt 缩合反应, 以中 等产率合成出二茂铁查尔酮化合物. 鉴于二茂铁查尔酮 的重要应用, 如何以温和的条件高产率地合成此类化合 物仍然是人们关注的方向. 本文研究乙酰基二茂铁与芳 香醛在离子液体(ILs)或相转移催化剂(PTCs)作用下进 行 Claisen-Schmidt 缩合反应.

\section{1 结果与讨论}

为了优化反应条件, 尝试乙酰基二茂铁(1)与苯甲 醛(2a)在各种条件下进行缩合, 结果例于表 1. 开始, 反 应用 $\mathrm{NaOH}$ 作为碱, 未加 ILs 和 PTCs, 在无水乙醇中进 行反应 $5 \mathrm{~h}$, 以 $72 \%$ 的产率得到化合物 $3 \mathrm{a}$ (Entry 1). 接 着尝试无溶剂条件, 加入 125 equiv. 的 $\mathrm{BMImPF}_{6}$ 反应 $7 \mathrm{~h}$ (Entry 2), 产率仅为 $51 \%$, 所以后续的反应均在无水乙 醇中进行. 当加入 0.75 equiv. 的 $\mathrm{NaOH}, 2.5$ equiv. 的 $\mathrm{BMImPF}_{6}$ (Entry 3) 时, 反应产率增加到 $82 \%$. 接下来通 过比较不同碱的催化情况来笁选所用的碱. 我们发现, 当使用 $\mathrm{K}_{2} \mathrm{CO}_{3}$ 或 $t-\mathrm{BuONa}$ 替代 $\mathrm{NaOH}$ 时(Entries 4, 5), 反 应产率降低, 因此选用 $\mathrm{NaOH}$ 为碱. 值得一提的是,
$\mathrm{NaOH}$ 为碱时, 离子液体 $\mathrm{BMImBF}_{4}$ 与 $\mathrm{BMImPF}_{6}$ 几乎起 同样的催化作用(Entry 6), 而当用阴离子 $\mathrm{Br}^{-} / \mathrm{Cl}^{-}$代替 $\mathrm{PF}_{6}^{-}$(表 1, Entries 7,8)或者用阳离子 $\mathrm{BPy}^{+}$替代 $\mathrm{BMIm}^{+}$ (Entries 9,10), 产率均有所下降.

接下来使用 PTCs 来替代 ILs, 我们欣喜的发现，当 用 $n-\mathrm{Bu}_{4} \mathrm{NPF}_{6}$ (Entry 11) 替代 BMImPF 时, $\mathbf{3 a}$ 的产率从 $82 \%$ 增加到 $94 \%$. 而用 $n-\mathrm{Bu}_{4} \mathrm{NBF}_{4}$ 或 $n-\mathrm{Bu}_{4} \mathrm{NBr}$ 替代 $n-\mathrm{Bu}_{4} \mathrm{NPF}_{6}$ (Entries 12,13) 时, 产率有所下降, 可见, PTCs 比 ILs 能更好的催化该反应，因此我们选用 $n-\mathrm{Bu}_{4} \mathrm{NPF}_{6}$ 作为催化剂. 后面的工作是用等物质的量的 $\mathbf{1}$ 与 $\mathbf{2 a}$ 反应, 确定 $\mathrm{NaOH}$ 与 $n-\mathrm{Bu}_{4} \mathrm{NPF}_{6}$ 的用量. 结果表 明，当 $\mathrm{NaOH}$ 的量由 0.75 equiv. 增加到 1 equiv. 时(Entry 14), 产率并没有提高; 而 $\mathrm{NaOH}$ 的量减少到 0.5 equiv. 时, 产率由 94\%降低到 75\% (Entry 15), 因此确定 $\mathrm{NaOH}$ 的量为 0.75 equiv. 接下来将 $n-\mathrm{Bu}_{4} \mathrm{NPF}_{6}$ 的量由 2.5 equiv. 降低到 2.0 equiv. (Entry 16), 产率由 94\%降低到 88\%, 而将 $n-\mathrm{Bu}_{4} \mathrm{NPF}_{6}$ 增加到 3.0 equiv. 时, 产率几乎没有提 高(Entry 17). 至此, 合成化合物 $\mathbf{3 a}$ 优化的反应条件已 经确立(Entry 11).

为了研究该反应条件的普适性，一系列芳香醛应用 于该反应，见表 2 . 结果表明，乙酰基二茂铁与取代的

表 1 反应条件的优化 ${ }^{a}$

Table 1 Optimisation of reaction conditions

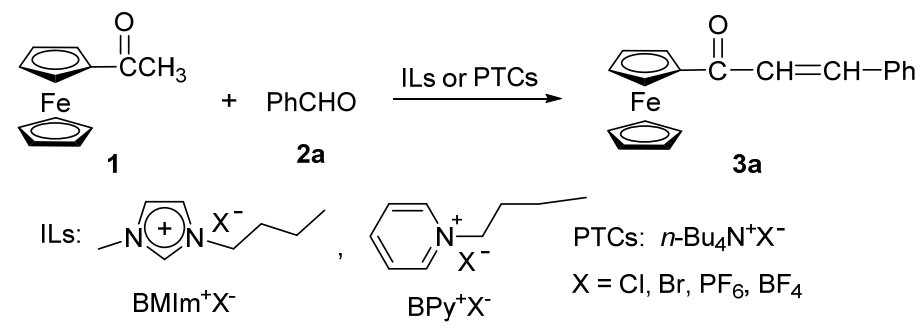

\begin{tabular}{|c|c|c|c|c|c|c|}
\hline Entry & ILs or PTCs/equiv. & Cation & Anion & Base (equiv.) & Time/h & Yield $^{b} / \%$ \\
\hline 1 & None & None & None & $\mathrm{NaOH}(1.0)$ & 5 & 72 \\
\hline 2 & $125^{c}$ & $\mathrm{BMIm}^{+}$ & $\mathrm{PF}_{6}^{-}$ & $\mathrm{NaOH}(1.0)$ & 7 & 51 \\
\hline 3 & 2.5 & $\mathrm{BMIm}^{+}$ & $\mathrm{PF}_{6}^{-}$ & $\mathrm{NaOH}(0.75)$ & 5 & 82 \\
\hline 4 & 2.5 & $\mathrm{BMIm}^{+}$ & $\mathrm{PF}_{6}^{-}$ & $\mathrm{K}_{2} \mathrm{CO}_{3}(0.75)$ & 10 & 61 \\
\hline 5 & 2.5 & $\mathrm{BMIm}^{+}$ & $\mathrm{PF}_{6}^{-}$ & $t$-BuONa $(0.75)$ & 5 & 75 \\
\hline 6 & 2.5 & $\mathrm{BMIm}^{+}$ & $\mathrm{BH}_{4}^{-}$ & $\mathrm{NaOH}(0.75)$ & 5 & 87 \\
\hline 7 & 2.5 & $\mathrm{BMIm}^{+}$ & $\mathrm{Br}^{-}$ & $\mathrm{NaOH}(0.75)$ & 6 & 68 \\
\hline 8 & 2.5 & $\mathrm{BMIm}^{+}$ & $\mathrm{Cl}^{-}$ & $\mathrm{NaOH}(0.75)$ & 7 & 63 \\
\hline 9 & 2.5 & $\mathrm{BPy}^{+}$ & $\mathrm{PF}_{6}^{-}$ & $\mathrm{NaOH}(0.75)$ & 5 & 75 \\
\hline 10 & 2.5 & $\mathrm{BPy}^{+}$ & $\mathrm{BH}_{4}^{-}$ & $\mathrm{NaOH}(0.75)$ & 5 & 70 \\
\hline 11 & 2.5 & $n-\mathrm{Bu}_{4} \mathrm{~N}^{+}$ & $\mathrm{PF}_{6}^{-}$ & $\mathrm{NaOH}(0.75)$ & 4 & 94 \\
\hline 12 & 2.5 & $n-\mathrm{Bu}_{4} \mathrm{~N}^{+}$ & $\mathrm{BH}_{4}^{-}$ & $\mathrm{NaOH}(0.75)$ & 4 & 88 \\
\hline 13 & 2.5 & $n-\mathrm{Bu}_{4} \mathrm{~N}^{+}$ & $\mathrm{Br}^{-}$ & $\mathrm{NaOH}(0.75)$ & 4 & 91 \\
\hline 14 & 2.5 & $n-\mathrm{Bu}_{4} \mathrm{~N}^{+}$ & $\mathrm{PF}_{6}^{-}$ & $\mathrm{NaOH}(1.0)$ & 4 & 94 \\
\hline 15 & 2.5 & $n-\mathrm{Bu}_{4} \mathrm{~N}^{+}$ & $\mathrm{PF}_{6}^{-}$ & $\mathrm{NaOH}(0.5)$ & 4 & 75 \\
\hline 16 & 2.0 & $n-\mathrm{Bu}_{4} \mathrm{~N}^{+}$ & $\mathrm{PF}_{6}^{-}$ & $\mathrm{NaOH}(0.75)$ & 4 & 88 \\
\hline 17 & 3.0 & $n-\mathrm{Bu}_{4} \mathrm{~N}^{+}$ & $\mathrm{PF}_{6}^{-}$ & $\mathrm{NaOH}(0.75)$ & 4 & 95 \\
\hline
\end{tabular}

${ }^{a}$ Molar ratio of compounds 1 and 2a is $1: 1$, in anhydrous $\mathrm{EtOH}$, at $35{ }^{\circ} \mathrm{C} ;{ }^{b}$ yield of isolated product; ${ }^{c}$ solvent free. 
苯甲醛及杂环芳香醛均能较高产率地得到二茂铁基查 尔酮. 我们还研究了醛中苯环的取代基效应对反应的影 响, 发现当苯环对位连有给电子的甲氧基时, 产率为 $87 \%$ (Entry 3), 而当苯环上连有拉电子的硝基时, 产率 高达 96\% (Entry 4). 这是因为 Claisen-Schmidt 缩合反应 属于亲核加成反应，给电子基团通过苯环的共轭作用传 递电子给醛基, 降低了醛基碳原子的亲核性, 不利于反 应的进行，而拉电子的硝基作用恰好相反，因此，产率 提高较为明显. 需要指出的是, 产物的 ${ }^{1} \mathrm{H}$ NMR 光谱数 据中, 从双键上两个质子的偶合常数为 $15.5 \mathrm{~Hz}$, 可推测 该反应得到的查尔酮均为 $E$-异构体, 因此该反应具有 较高的立体选择性.

\section{表 2 二茂铁基查尔酮的制备 ${ }^{a}$}

Table 2 Preparation of ferrocenylchalcone

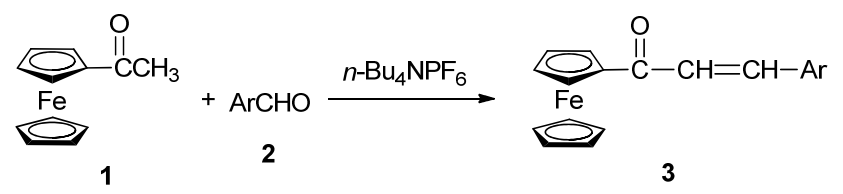

$\mathrm{Ar}=\mathrm{C}_{6} \mathrm{H}_{5}(\mathbf{2 a}), p-\mathrm{Cl}-\mathrm{C}_{6} \mathrm{H}_{4}(\mathbf{2 b}), p-\mathrm{CH}_{3} \mathrm{O}-\mathrm{C}_{6} \mathrm{H}_{4}(\mathbf{2} \mathbf{c}), p-\mathrm{NO}_{2}-\mathrm{C}_{6} \mathrm{H}_{4}(\mathbf{2 d})$, $m-\mathrm{NO}_{2}-\mathrm{C}_{6} \mathrm{H}_{4}$ (2e), 2-pyridyl (2f), 2-furyl (2g), 2-thienyl (2h)

\begin{tabular}{cccc}
\hline Entry & Compund & Time/h & Yield $^{b} \%$ \\
\hline 1 & 3a & 4 & 94 \\
2 & 3b & 5 & 89 \\
3 & 3c & 8 & 87 \\
4 & 3d & 4 & 96 \\
5 & 3e & 4 & 98 \\
6 & $\mathbf{3 f}$ & 6 & 93 \\
7 & $\mathbf{3 g}$ & 6 & 96 \\
8 & $\mathbf{3 h}$ & 4 & 90 \\
\hline
\end{tabular}

${ }^{a}$ Molar ratio of compounds $1,2, n-\mathrm{Bu}_{4} \mathrm{NPF}_{6}$ and $\mathrm{NaOH}$ is $1: 1: 2.5: 0.75$, in anhydrous $\mathrm{EtOH}$, at $35{ }^{\circ} \mathrm{C} ;{ }^{b}$ Yield of isolated product.

\section{2 结论}

乙酰基二茂铁与苯甲醛、取代苯甲醛以及杂环芳香 醛在离子液体或相转移催化剂作用下进行 ClaisenSchmidt 缩合, 高产率地合成一系列二茂铁基查尔酮. 其中, 相转移催化剂能更有效地催化该反应, 部分查尔 酮产率高达 $98 \%$. 所有反应都在无水乙醇溶液, $35{ }^{\circ} \mathrm{C}$ 条 件下进行, 优化后的乙酰基二茂铁：芳香醛：四丁基六 氟磷酸铵: $\mathrm{NaOH}$ 的物质的量比为 $1: 1: 2.5: 0.75$. 由 于二茂铁基查尔酮在有机化学、药物化学以及材料化学 日益重要, 这种高产率合成二茂铁基查尔酮的方法具有 重要意义.

\section{3 实验部分}

\section{1 仪器与试剂}

NEXUS-670 傅里叶变换红外光谱仪, $\mathrm{KBr}$ 压片;
Avance 500 Bruker (500 MHz)核磁共振仪, 以 TMS 为内 标; XT7-4 型熔点仪. 所用试剂均为市售分析纯试剂, 溶剂的干燥按常规方法进行.

\section{2 实验方法}

在 $100 \mathrm{~mL}$ 圆底瓶中, 依次加入 $n-\mathrm{Bu}_{4} \mathrm{NPF}_{6}(1.935 \mathrm{~g}$, $5 \mathrm{mmol}), \mathrm{NaOH}(0.06 \mathrm{~g}, 1.5 \mathrm{mmol})$ 和无水 $\mathrm{EtOH}(10 \mathrm{~mL})$, 然后于 $35{ }^{\circ} \mathrm{C}$ 滴加化合物 $1(0.456 \mathrm{~g}, 2 \mathrm{mmol})$ 溶于 $8 \mathrm{~mL}$ 无水 $\mathrm{EtOH}$ 的溶液, 搅拌 $10 \mathrm{~min}$. 接着滴加化合物 2 (2 $\mathrm{mmol}$ )溶于 $2 \mathrm{~mL}$ 无水 $\mathrm{EtOH}$, 在 $35{ }^{\circ} \mathrm{C}$ 继续摚拌. 薄板层 析监测反应进程. 反应结束, 室温放置过夜, 有沉淀产 生, 抽滤, 水洗、冰冷的无水乙醇洗涤, 乙醇重结晶得到 纯净的化合物 $\mathbf{3}$.

1-二茂铁基-3-苯基-2-丙烯-1-酮(3a): 橘红色固体, m.p. 138 $139{ }^{\circ} \mathrm{C}$ (lit. $\left.{ }^{[24]} 141{ }^{\circ} \mathrm{C}\right) ;{ }^{1} \mathrm{H}$ NMR $(500 \mathrm{MHz}$, $\left.\mathrm{CDCl}_{3}\right) \delta: 7.80(\mathrm{~d}, J=15.5 \mathrm{~Hz}, 1 \mathrm{H}, \mathrm{CH}=\mathrm{CH}), 7.66 \sim 7.64$ $\left(\mathrm{m}, 2 \mathrm{H}, \mathrm{C}_{6} \mathrm{H}_{5}\right), 7.45 \sim 7.41\left(\mathrm{~m}, 3 \mathrm{H}, \mathrm{C}_{6} \mathrm{H}_{5}\right), 7.16(\mathrm{~d}, J=15.5$ $\mathrm{Hz}, 1 \mathrm{H}, \mathrm{CH}=\mathrm{CH}), 4.92$ (s, 2H, Fc), 4.60 (s, 2H, Fc), 4.22 (s, 5H, Fc); IR (KBr) v: 3086, 1649, 1597, 1454, 1238, $1077,995,822,762,502 \mathrm{~cm}^{-1}$. Anal. calcd for $\mathrm{C}_{19} \mathrm{H}_{16} \mathrm{FeO}$ : C 72.18, H 5.10; found C 72.27, H 5.15.

1-二茂铁基-3-(4-氯苯基)-2-丙烯-1-酮(3b): 橘红色 固体, m.p. $159 \sim 161{ }^{\circ} \mathrm{C}$ (lit. ${ }^{[21]} 161 \sim 162{ }^{\circ} \mathrm{C}$ ); ${ }^{1} \mathrm{H}$ NMR $\left(500 \mathrm{MHz}, \mathrm{CDCl}_{3}\right) \delta: 7.73(\mathrm{~d}, J=15.5 \mathrm{~Hz}, 1 \mathrm{H}, \mathrm{CH}=\mathrm{CH})$, 7.57 (d, $\left.J=7.0 \mathrm{~Hz}, 2 \mathrm{H}, \mathrm{C}_{6} \mathrm{H}_{4}\right), 7.39$ (d, J=7.0 Hz, 2H, $\left.\mathrm{C}_{6} \mathrm{H}_{4}\right), 7.10(\mathrm{~d}, J=15.5 \mathrm{~Hz}, 1 \mathrm{H}, \mathrm{CH}=\mathrm{CH}), 4.91(\mathrm{~s}, 2 \mathrm{H}$, $\mathrm{Fc}), 4.60(\mathrm{~s}, 2 \mathrm{H}, \mathrm{Fc}), 4.21(\mathrm{~s}, 5 \mathrm{H}, \mathrm{Fc})$; IR (KBr) v: 3130, 1651, 1594, 1401, 1082, 828, $486 \mathrm{~cm}^{-1}$. Anal. calcd for $\mathrm{C}_{19} \mathrm{H}_{15} \mathrm{ClFeO}$ : C 65.09, H 4.31; found C 65.26, H 4.54.

1-二茂铁基-3-(4-甲氧基苯基)-2-丙烯-1-酮(3c): 橘 红色固体, m.p. $153 \sim 154{ }^{\circ} \mathrm{C}$ (lit. ${ }^{[24]} 149{ }^{\circ} \mathrm{C}$ ); ${ }^{1} \mathrm{H}$ NMR $\left(500 \mathrm{MHz} \mathrm{CDCl}_{3}\right) \delta: 7.75(\mathrm{~s}, 1 \mathrm{H}, \mathrm{CH}=\mathrm{CH}), 7.61(\mathrm{~s}, 2 \mathrm{H}$, $\left.\mathrm{C}_{6} \mathrm{H}_{4}\right), 6.95\left(\mathrm{~s}, 3 \mathrm{H}, \mathrm{CH}=\mathrm{CH}, \mathrm{C}_{6} \mathrm{H}_{4}\right), 4.91(\mathrm{~s}, 2 \mathrm{H}, \mathrm{Fc}), 4.56$ (s, 2H, Fc), 4.21 (s, 5H, Fc), $3.86\left(\mathrm{~s}, 3 \mathrm{H}, \mathrm{CH}_{3}\right)$; IR (KBr) v: 3130, 1648, 1582, 1401, 1259, 1165, 1049, 1173, 1079, $828,503 \mathrm{~cm}^{-1}$. Anal. calcd for $\mathrm{C}_{20} \mathrm{H}_{18} \mathrm{FeO}_{2}$ : C 69.39, $\mathrm{H}$ 5.24; found C 68.93, H 5.30.

1-二茂铁基-3-(4-硝基苯基)-2-丙烯-1-酮(3d)：橘红 色固体, m.p. 190 191 ${ }^{\circ} \mathrm{C}$ (lit. $\left.{ }^{[21]} 192{ }^{\circ} \mathrm{C}\right) ;{ }^{1} \mathrm{H}$ NMR $(500$ $\left.\mathrm{MHz}, \mathrm{CDCl}_{3}\right) \delta: 7.76(\mathrm{~s}, 1 \mathrm{H}, \mathrm{CH}=\mathrm{CH}), 7.61(\mathrm{~s}, 2 \mathrm{H}$, $\left.\mathrm{C}_{6} \mathrm{H}_{4}\right), 7.10 \sim 6.95\left(\mathrm{~m}, 3 \mathrm{H}, \mathrm{CH}=\mathrm{CH}, \mathrm{C}_{6} \mathrm{H}_{4}\right), 4.91(\mathrm{~s}, 2 \mathrm{H}$, $\mathrm{Fc}), 4.56$ (s, 2H, Fc), 4.21 (s, 5H, Fc); IR (KBr) v: 3086, $1647,1581,1510,1258,1173,1079,827,503 \mathrm{~cm}^{-1}$. Anal. calcd for $\mathrm{C}_{19} \mathrm{H}_{15} \mathrm{FeNO}_{3}$ : C 63.18, H 4.19, N 3.88; found $\mathrm{C}$ 63.33, H 4.15, N 3.62.

1-二茂铁基-3-(3-硝基苯基)-2-丙烯-1-酮(3e)：橘红 
色固体, m.p. $171 \sim 173{ }^{\circ} \mathrm{C}$ (lit. ${ }^{[22]} 171 \sim 172{ }^{\circ} \mathrm{C}$ ); ${ }^{1} \mathrm{H}$ NMR (500 MHz, $\left.\mathrm{CDCl}_{3}\right) \delta: 8.51\left(\mathrm{~s}, 1 \mathrm{H}, \mathrm{C}_{6} \mathrm{H}_{4}\right), 8.25(\mathrm{~s}$, $\left.1 \mathrm{H}, \mathrm{C}_{6} \mathrm{H}_{4}\right), 7.91\left(\mathrm{~s}, 1 \mathrm{H}, \mathrm{C}_{6} \mathrm{H}_{4}\right), 7.80(\mathrm{~s}, 1 \mathrm{H}, \mathrm{CH}=\mathrm{CH}), 7.61$ (s, $\left.1 \mathrm{H}, \mathrm{C}_{6} \mathrm{H}_{4}\right), 7.22$ (s, 1H, $\left.\mathrm{CH}=\mathrm{CH}\right), 4.94$ (s, 2H, Fc), 4.65 (s, 2H, Fc), 4.23 (s, 5H, Fc); IR (KBr) v: 3013, 1650, $1603,525,1400,1000,722,484 \mathrm{~cm}^{-1}$. Anal. calcd for $\mathrm{C}_{19} \mathrm{H}_{15} \mathrm{FeNO}_{3}$ : C 63.18, H 4.19, N 3.88; found $\mathrm{C} 62.83, \mathrm{H}$ 4.24, N 3.95 .

1-二茂铁基-3-(2-吡啶基)-2-丙烯-1-酮(3f): 红棕色 固体, m.p. $151 \sim 153{ }^{\circ} \mathrm{C}$ (lit. ${ }^{[21]} 152 \sim 153{ }^{\circ} \mathrm{C}$ ); ${ }^{1} \mathrm{H}$ NMR $\left(500 \mathrm{MHz}, \mathrm{CDCl}_{3}\right) \delta: 8.70(\mathrm{~s}, 1 \mathrm{H}$, pyridinyl), 7.75 (d, $J=$ $15.0 \mathrm{~Hz}, 1 \mathrm{H}, \mathrm{CH}=\mathrm{CH}), 7.71$ (s, 1H, pyridinyl), 7.66 (d, $J=15.0 \mathrm{~Hz}, 1 \mathrm{H}, \mathrm{CH}=\mathrm{CH}), 7.46(\mathrm{~s}, 1 \mathrm{H}$, pyridinyl), 7.30 7.27 (s, 1H, pyridinyl), 4.97 (s, 2H, Fc), 4.61 (s, 2H, Fc), 4.23 (s, 5H, Fc); IR (KBr) v: 3127, 1652, 1597, 1461, 1401, 1241, 1081, 988, 827, $546 \mathrm{~cm}^{-1}$. Anal. calcd for $\mathrm{C}_{18} \mathrm{H}_{15}$ FeNO: $\mathrm{C}$ 68.16, $\mathrm{H} 4.77, \mathrm{~N} 4.42$; found $\mathrm{C} 67.71, \mathrm{H}$ 4.83, N 4.03.

1-二茂铁基-3-(2-呋喃基)-2-丙烯-1-酮(3g): 红棕色 固体, m.p. $155 \sim 156{ }^{\circ} \mathrm{C}$ (lit. $\left.{ }^{[24]} 152{ }^{\circ} \mathrm{C}\right)$; ${ }^{1} \mathrm{H}$ NMR $(500$ $\left.\mathrm{MHz}, \mathrm{CDCl}_{3}\right) \delta 7.55(\mathrm{~d}, J=15.0 \mathrm{~Hz}, 1 \mathrm{H}, \mathrm{CH}=\mathrm{CH}), 7.53$ (s, 1H, furanyl), $7.00(\mathrm{~d}, J=15.0 \mathrm{~Hz}, 1 \mathrm{H}, \mathrm{CH}=\mathrm{CH}), 6.68$ (s, 1H, furanyl), $6.51(\mathrm{~s}, 1 \mathrm{H}$, furanyl), $4.90(\mathrm{~s}, 2 \mathrm{H}, \mathrm{Fc})$, 4.57 (s, 2H, Fc), 4.21 (s, 5H, Fc); IR (KBr) v: 3087, 1651, 1592, 1301, 1282, 1078, 1015, 746, $505 \mathrm{~cm}^{-1}$. Anal. calcd for $\mathrm{C}_{17} \mathrm{H}_{14} \mathrm{FeO}_{2}$ : C 66.70, H 4.61; found C 66.42, H 5.13.

1-二茂铁基-3-(2-噻吩基)-2-丙烯-1-酮(3h): 红棕色 固体, m.p. $145 \sim 147{ }^{\circ} \mathrm{C}$ (lit. $\left.{ }^{[24]} 146{ }^{\circ} \mathrm{C}\right)$; ${ }^{1} \mathrm{H}$ NMR $(500$ $\left.\mathrm{MHz} \mathrm{CDCl}_{3}\right) \delta: 7.91(\mathrm{~d}, J=13.5 \mathrm{~Hz}, 1 \mathrm{H}, \mathrm{CH}=\mathrm{CH}), 7.53$ (s, 1H, furanyl), $6.92(\mathrm{~d}, J=13.5 \mathrm{~Hz}, 1 \mathrm{H}, \mathrm{CH}=\mathrm{CH}), 6.68$ (s, 1H, furanyl), $6.51(\mathrm{~s}, 1 \mathrm{H}$, furanyl), $4.90(\mathrm{~s}, 2 \mathrm{H}, \mathrm{Fc})$, 4.57 (s, 2H, Fc), 4.21 (s, 5H, Fc); IR (KBr) v: 3106, 1643, 1583, 1458, 1083, 1001, 857, 706, $503 \mathrm{~cm}^{-1}$. Anal. calcd for $\mathrm{C}_{17} \mathrm{H}_{14} \mathrm{FeOS}$ : C 63.37, H 4.38; found C 63.51, H 4.55.

\section{辅助材料(Supporting Information) 化合物 $\mathbf{3 a} \sim \mathbf{3 h}$ 的} ${ }^{1} \mathrm{H}$ NMR 谱图. 这些材料可以免费从本刊网站(http:// sioc-journal.cn/)上下载.

\section{References}

[1] Patil, P. S.; Dharmaprakash, S. M.; Ramakrishna, K.; Fun, H. K. J. Cryst. Growth 2007, 303, 520.

[2] Zhao, H.; Zhu, X.; Wang, D.; Chen, S.; Bian, Z. Aust. J. Chem. 2015, 68, 1035.

[3] Avila, H.; Smania, E.; Monache, F.; Junior, A. Bioorg. Med. Chem. 2008, 16, 9790.

[4] Niu, C.; Li, G.; Tuerxuntayi, A.; Aisa, H. A. Chin. J. Chem. 2015, $33,486$.

[5] Prasath, R.; Bhavana, P.; Ng, S. W.; Tiekink, E. R. T. J. Organomet. Chem. 2013, 726, 62.

[6] Attar, S.; O'Brien, Z.; Alhaddad, H.; Golden, M. L.; Calderón-Urrea, A. Bioorg. Med. Chem. 2011, 19, 2055.

[7] Wu, X.; Tiekink, E. R. T.; Kostetski, I.; Kocherginsky, N.; Tan, A. L. C.; Khoo, S. B.; Wilairat, P.; Go, M. L. Eur. J. Pharm. Sci. 2006, $27,175$.

[8] Wang, D.; Zhu, X. Y.; Zhao, H. Y.; Bian, Z. X. Chin. J. Org. Chem. 2015, 35, 1131 (in Chinese). (王栋, 朱学友, 赵海英, 边占喜, 有机化学, 2015, 35, 1131.)

[9] Yang, J. M.; Ji, S. J.; Gu, D. G.; Shen, Z. L.; Wang, S. Y. J. Organomet. Chem. 2005, 690, 2989.

[10] Parveen, H.; Hayat, F.; Salahuddin, A.; Azam, A. Eur. J. Med. Chem. 2010, 45, 3497.

[11] Gong, Z. L.; Xie, Y. S; Zhao, B. X.; Lv, H. S.; Liu, W. Y.; Zheng, L. W.; Lian, S. J. Fluoresc. 2011, 21, 355.

[12] Bukhari, S. N. A.; Jasamai, M.; Jantan, I.; Ahmad, W. Mini-Rev. Org. Chem. 2013, 10, 73.

[13] Fang, D.; Cheng, J.; Fei, Z.; Gong, K.; Liu, Z. Catal. Commun. 2008, 9, 1924.

[14] Shibata, K.; Katsuyama, I.; Matsui, M.; Muramatsu, H. Bull. Chem. Soc. Jpn. 1990, 63, 3710.

[15] Erasmus, E. Inorg. Chim. Acta 2011, 378, 95.

[16] Abashev, G. G.; Antuf'eva, A. D.; Bushueva, A. Y.; Kudryavtsev, P. G.; Osorgina, I. V.; Syutkin, R. V.; Shklyaeva, E. V. Russ. J. Appl. Chem. 2010, 83, 1435.

[17] Muller, T. J.; Conradie, J.; Erasmus, E. Polyhedron 2012, 33, 257.

[18] Srivastava, Y. K. Rasayan J. Chem. 2008, 1, 884.

[19] Liu, Y. T.; Lian, G. D.; Yin, D. W.; Su, B. J. Res. Chem. Intermed. 2012, 38, 1043.

[20] Liu, Y. T.; Feng, L.; Yin, D. W. Res. Chem. Intermed. 2013, 39, 2971.

[21] Ji, S. J.; Wang, S. Y.; Shen, Z. L.; Zhou, M. F. Chin. Chem. Lett. 2003, 14, 1246.

[22] Liu, W.; Xu, Q.; Chen, B.; Ma, Y. Synth. Commun. 2002, 32, 171.

[23] Salisova, M.; Puciova, M.; Postnov, U. N.; Toma, S. Chem. Papers 1990, 44, 201.

[24] Villemin, D.; Martin, B.; Puciova, M.; Toma, S. J. Organomet. Chem. 1994, 484, 27. 